\title{
Auditory brain stem responses evoked by lateralized clicks: is lateralization extracted in the human brain stem?
}

\author{
Helmut Riedel, Birger Kollmeier * \\ AG Medizinische Physik, Universität Oldenburg, D-26111 Oldenburg, Germany
}

Received 22 March 2001; accepted 3 August 2001

\begin{abstract}
The dependence of binaurally evoked auditory brain stem responses and the binaural difference potential on simultaneously presented interaural time and level differences is investigated in order to assess the representation of stimulus lateralization in the brain stem. Auditory brain stem responses to binaural click stimuli with all combinations of three interaural time and three interaural level differences were recorded from 12 subjects and 4 channels. The latency of Jewett wave V is shortest for zero interaural time difference and longest for the trading stimuli. The amplitude of wave $\mathrm{V}$ is largest for centrally perceived stimuli, i.e., the diotic and trading stimuli, and smallest for the most laterally perceived stimuli. The latency of the most prominent peak of the binaural difference potential DN1 mainly depends on the interaural time difference. The amplitude of the components of the binaural difference potential, DP1-DN1, depends similarly on stimulus conditions as wave V amplitude in the case of the binaural stimuli: smallest amplitudes are found for the most lateral stimuli and largest amplitudes for central stimuli. The results demonstrate that interaural level and time differences are not processed independently. This supports the hypothesis that directional information in humans is already extracted and represented at the level of the brain stem. (C) 2002 Elsevier Science B.V. All rights reserved.
\end{abstract}

Key words: Auditory brain stem response; Binaural difference potential; Interaural time difference; Interaural level difference; Lateralization

\section{Introduction}

From psychoacoustical studies it is known that the most important cues for directional hearing are the interaural time difference (ITD) and the interaural level difference (ILD) (Feddersen et al., 1957; Mills, 1958, 1960; Colburn and Durlach, 1978). Presenting a stimulus with an ITD and an ILD that point towards the

\footnotetext{
* Corresponding author. Tel.: +49-441-7985466; Fax: +49-441-7983698.

E-mail address: birger.kollmeier@uni-oldenburg.de (B. Kollmeier).
}

Abbreviations: ABR, auditory brain stem response; ACT, acoustic crosstalk; $\mathrm{BD}$, binaural difference potential; $\mathrm{CN}$, cochlear nucleus; EE, excitatory-excitatory; IC, inferior colliculus; IE, inhibitoryexcitatory; ILD, interaural level difference; ITD, interaural time difference; LL, lateral lemniscus; MER, middle ear reflex; MNTB, medial nucleus of the trapezoid body; nHL, normal hearing level; NLL, nuclei of the lateral lemniscii; SNR, signal-to-noise ratio; SO, superior olive; SPL, sound pressure level; TIR, time-intensity-trading ratio same direction ('synergistic presentation') leads to a further lateralized perception than presenting the same respective ITD or ILD alone. In contrast, the 'antagonistic presentation', when ILD and ITD point to opposite lateral positions, results in more central percepts. The relation between an ILD and the ITD that causes the same lateralization is commonly expressed in terms of the time-intensity-trading ratio (TIR). It can be measured either by subjectively matching the respective lateralization produced by ILD and ITD or by trading ILD against ITD resulting in a centered image. TIRs were measured for a variety of stimuli and levels (e.g., Durlach and Colburn, 1978; Nordby et al., 1982; Furst et al., 1985; McPherson and Starr, 1995; Damaschke et al., 2000).

From neuroanatomical studies it is known that left and right auditory afferent fibers first intersect in the superior olive (SO) in the brain stem (Nieuwenhuys et al., 1988). Neurophysiological studies in animals demonstrate binaural processing in cells of the $\mathrm{SO}$ and sub- 
sequent stations of the auditory brain stem, namely the nuclei of the lateral lemnisci (NLL) and the inferior colliculus (IC) (Semple and Aitkin, 1989; Achor and Starr, 1980; Caird and Klinke, 1983; Caird et al., 1985; Yin and Chan, 1990; Popper and Fay, 1992; Joseph and Hyson, 1993; Gummer and Zenner, 1996; van Adel et al., 1999).

In evoked response studies binaural processing or binaural interaction is assessed in terms of the binaural difference potential (BD). It is defined as the difference between the potential obtained with binaural stimulation and the sum of the potentials obtained with monaural stimulation, symbolically $\mathrm{BD}=\mathrm{B}-(\mathrm{L}+\mathrm{R})$. Any significant deviation from $\mathrm{BD}=0$ is understood as hint to some non-linearity, i.e., a functional coupling of left and right signals. The BD was analyzed in a number of studies, often as a function of ILD and ITD (Dobie and Berlin, 1979; Dobie and Norton, 1980; Ainslie and Boston, 1980; Levine, 1981; Wrege and Starr, 1981; Gerull and Mrowinski, 1984; KellyBallweber and Dobie, 1984; Furst et al., 1985; Ito et al., 1988; McPherson et al., 1989; Jones and van der Poel, 1990; McPherson and Starr, 1995; Jiang, 1996; Cone-Wesson et al., 1997; Brantberg et al., 1999a,b). Using 2000 sweeps per stimulus condition Gerull and Mrowinski (1984) contended the null hypothesis $\mathrm{BD}=0$, i.e., that the binaural response $\mathrm{B}$ can be perfectly predicted by the sum of the monaural responses $\mathrm{L}+\mathrm{R}$, supporting the view of two independent channels. Ainslie and Boston (1980), also using 2000 sweeps, found a non-vanishing BD but explained it by acoustic crosstalk (ACT). A thorough investigation of possible artifacts resulting in an artificial BD was performed by Levine (1981). He described two possible sources of artifacts: ACT and the middle ear reflex (MER). These have to be ruled out before associating a measured BD with neural processes.

Furst et al. (1985) suggested that the first major peak in the $\mathrm{BD}, \mathrm{DN} 1$ or $\beta$ in their nomenclature, is a physiological correlate of the categorial percept of binaural fusion. DN1 was present up to ITDs of $1 \mathrm{~ms}$ with a relatively constant amplitude, but undetectable for ITDs longer than $1.2 \mathrm{~ms}$. With increasing ILD, DN1 amplitude decreased gradually and became undetectable for ILDs greater than $30 \mathrm{~dB}$. Brantberg et al. (1999a) studied DN1 as a function of the ITD and found approximately constant amplitudes for ITDs up to $1 \mathrm{~ms}$. In contrast, McPherson and Starr (1995) reported that the DN1 component gradually decreased with increasing ILD and ITD, and became undetectable for ILD $>16 \mathrm{~dB}$ and ITD $>1.6 \mathrm{~ms}$. They stated an inverse correlation between DN1 amplitude and the psychophysical lateralization (introduced by either ILD or ITD).

The major problem of measuring the $\mathrm{BD}$ is its poor signal-to-noise ratio (SNR). The BD is about an order of magnitude smaller than binaural responses, and its residual noise is about twice as large as that of a directly measured potential due to the calculation of sums and differences. Sweep numbers of about 2000, which are generally sufficient in auditory brain stem response (ABR) recordings, may result in a poor SNR of the BD. Hence, for a reliable detection of BD components high-quality recordings and a precise judgement of the SNR are required. In the present study, this was accomplished by storing all unfiltered single sweep to disk, offline linear phase filtering and iterative, weighted averaging. The residual noise was calculated as the standard error of the mean over all filtered single sweeps, or more precisely, as the rms value of the time dependent standard error $\sigma(t)$ for every channel, stimulus condition and subject (Riedel et al., 2001). This is in contrast to other studies, where the residual noise was estimated from the difference of two averages recorded, (e.g., Wrege and Starr, 1981) or from the average in the prestimulus interval, (e.g., Furst et al., 1985).

In all studies known to the authors, only the effect of a single parameter (either ILD or ITD) on the BD was studied without relating it to the psychophysical effect of lateralization which arises from a combination of both parameters. The aim of the present study therefore is to analyze the dependence of ABRs and BD on psychophysical lateralization if not only a single cue, ILD or ITD, is presented, but also for synergistic and trading stimulus configurations. The question is whether stimuli with similar lateralization evoke similar responses. If so, this would imply that a representation of the laterality of a stimulus would already exist at brain stem level, and that ILD and ITD were not processed independently.

\section{Materials and methods}

\subsection{Subjects}

Twelve subjects from the staff of the University of Oldenburg (three females, nine males) between the ages of 25 and 36 participated voluntarily in this study. They had no history of audiological or neurological problems and were classified as normal hearing by routine audiometry. The audiometric loss was less than 10 $\mathrm{dB}$ for frequencies below $4 \mathrm{kHz}$ and less than $15 \mathrm{~dB}$ for the higher frequencies.

\subsection{Stimuli}

Rarefaction click stimuli were produced by applying rectangular voltage pulses of $100-\mu$ s duration to Etymotic Research ER-2 insert earphones. The time inter- 
val between the onsets of two successive stimuli was chosen to vary randomly and equally distributed between 62 and $72 \mathrm{~ms}$, yielding an average stimulation rate of approximately $15 \mathrm{~Hz}$. A 700-ms segment of the click train comprising 11 clicks was used to determine the thresholds in quiet. They were measured three times by all subjects with a three-alternative-forcedchoice-method in conjunction with a two-down-oneup algorithm for both ears and averaged over runs, subjects and ears. The threshold level - referred to as $0 \mathrm{~dB}$ normal hearing level (nHL) - corresponds to 39 $\mathrm{dB}$ peak equivalent sound pressure level (p.e. SPL) ${ }^{1}$. The standard deviation of the individual thresholds from the averaged threshold was $3 \mathrm{~dB}$. For reference also the thresholds for single clicks were determined. On average, single-click thresholds were $5 \mathrm{~dB}$ higher than click train thresholds.

Fifteen stimulus conditions were tested, nine binaural and six monaural. The monaural clicks were presented at the levels 53, 59 and $65 \mathrm{~dB} \mathrm{nHL}$ and are denoted as $\mathrm{L}-\mathrm{m}, \mathrm{L} 0 \mathrm{~m}, \mathrm{~L}+\mathrm{m}$ and $\mathrm{R}-\mathrm{m}, \mathrm{R} 0 \mathrm{~m}, \mathrm{R}+\mathrm{m}$ for monaural left and right stimulation, respectively. The binaural stimuli, see Fig. 1, were the nine possible combinations of three ITDs $(-0.4,0$ and $0.4 \mathrm{~ms})$ and three ILDs $(-12,0$ and $12 \mathrm{~dB})$.

The binaural stimuli are named as follows: the first letter refers to the perceived lateralization of the stimuli: ' $L$ ' for left, ' $C$ ' for center and ' $R$ ' for right. The second and third characters (' - ', ' 0 ' and ' + ') are used to specify the ILD and ITD, respectively. For example, for diotic stimulation to both ears a click at $59 \mathrm{~dB} \mathrm{nHL}$ was presented simultaneously. This stimulus $\mathrm{C} 00$ is found in the center of the diagram $(\mathrm{ILD}=\mathrm{ITD}=0$ ). The stimulus $\mathrm{R}+0$ in the middle of the top row has zero ITD, but is lateralized to the right due to its positive ILD. On the other hand, the stimulus $\mathrm{R} 0+$ at the right of the middle row has zero ILD, but is also lateralized to the right due to its positive ITD. The arrows in Fig. 1 point into the approximate direction of the lateralization of the stimuli. Both an ITD of $0.4 \mathrm{~ms}$ and an ILD of $12 \mathrm{~dB}$ cause a strong, but not extreme lateralization of about $70^{\circ}$ (Furst et al., 1985; McPherson and Starr, 1995). A stronger, almost complete lateralization is produced by the synergistic stimuli $\mathrm{L}--$ and $\mathrm{R}++$ whose ILDs and ITDs point into the same direction. In contrast, the stimuli $\mathrm{C}+-$ and $\mathrm{C}-+$ refer to the antagonistic situation: ILD and ITD act in opposite direction resulting in a centered image. In the lower left corner of each subplot in Fig. 1, the respective binaural stimulus is depicted schematically. In the

\footnotetext{
1 A sinusoid of frequency $1 \mathrm{kHz}$ with the same peak-to-peak-amplitude showed $39 \mathrm{~dB}$ SPL in a Brüel and Kjær (B\&K) amplifier type 2610. The calibration was performed using a half-inch microphone (B\&K 4134) with an artificial ear and a preamplifier (B\&K 2669).
}

ITD-ILD plane lines of equal lateralization are the diagonal dotted lines. We used identical stimuli for all subjects for better comparability of the results. Comparatively large values for the ITD and the ILD were used to obtain as large differences in the evoked potentials for the different stimulus conditions as possible without leaving the physiological range.

\subsection{Electrodes}

For the $\mathrm{ABR}$ recordings $\mathrm{Ag} / \mathrm{AgCl}$-electrodes were used. The four active channels were placed at the left (A1) and right (A2) mastoid and the parieto-occipital positions PO9 and PO10 according to the extended 10 20 system (Jasper, 1957; Sharbrough et al., 1991). The common reference electrode was placed at the vertex $(\mathrm{Cz})$, the ground electrode at the forehead (Fpz). Electrode impedances were measured at a test signal frequency of $30 \mathrm{~Hz}$ and brought well below $5 \mathrm{k} \Omega$, common values were $2-3 \mathrm{k} \Omega$. Since DC recordings were performed, the criteria for a good contact between electrodes and skin were both a low impedance and a vanishing voltage drift seen in the raw EEG signal.

\subsection{Recordings}

During the ABR recordings, subjects lay in a sound insulated and electrically shielded room. They were instructed to relax and lie as comfortably as possible. ABR were recorded with a DC-coupled differential amplifier (Synamps 5803). Inside the shielded room the EEG was preamplified by a factor 150 , further amplified by the main amplifier by a factor 33 resulting in a total amplification of $74 \mathrm{~dB}$. The voltage resolution was approximately $16.8 \mathrm{nV} / \mathrm{bit}$. The sweeps were filtered by an analog antialiasing-lowpass with a corner frequency of $2 \mathrm{kHz}$, digitized with $10 \mathrm{kHz}$ sampling rate and 16 bit resolution, and stored to hard disk.

During the recording the artifact rejection level was set to $\pm 500 \mu \mathrm{V}$, since filtering, artifact analysis and averaging was done offline. The clipping level of the DA-converters was $\pm 550 \mu \mathrm{V}$. The recording interval comprised 400 samples in the time interval from -15 to $25 \mathrm{~ms}$ relative to stimulus onset. For the dichotic stimuli the leading click was defined as stimulus onset.

10000 single sweeps for all of the 15 stimuli were recorded in the following manner: The five stimuli belonging to a certain ILD (a row in Fig. 1, e.g., $\mathrm{L}-\mathrm{m}$, $\mathrm{C}+-, \mathrm{R}+0, \mathrm{R}++$ and $\mathrm{R}+\mathrm{m}$ ) were presented in random order on a sweep-by-sweep basis. One run consisting of 12500 stimuli, 2500 of each type, lasted approximately $14 \mathrm{~min}$. After each run the impedances were checked, and adjusted if necessary. Afterwards the runs belonging to the two other ILDs were recorded. Totally, 12 runs (four repetitions of the three runs) were carried out 


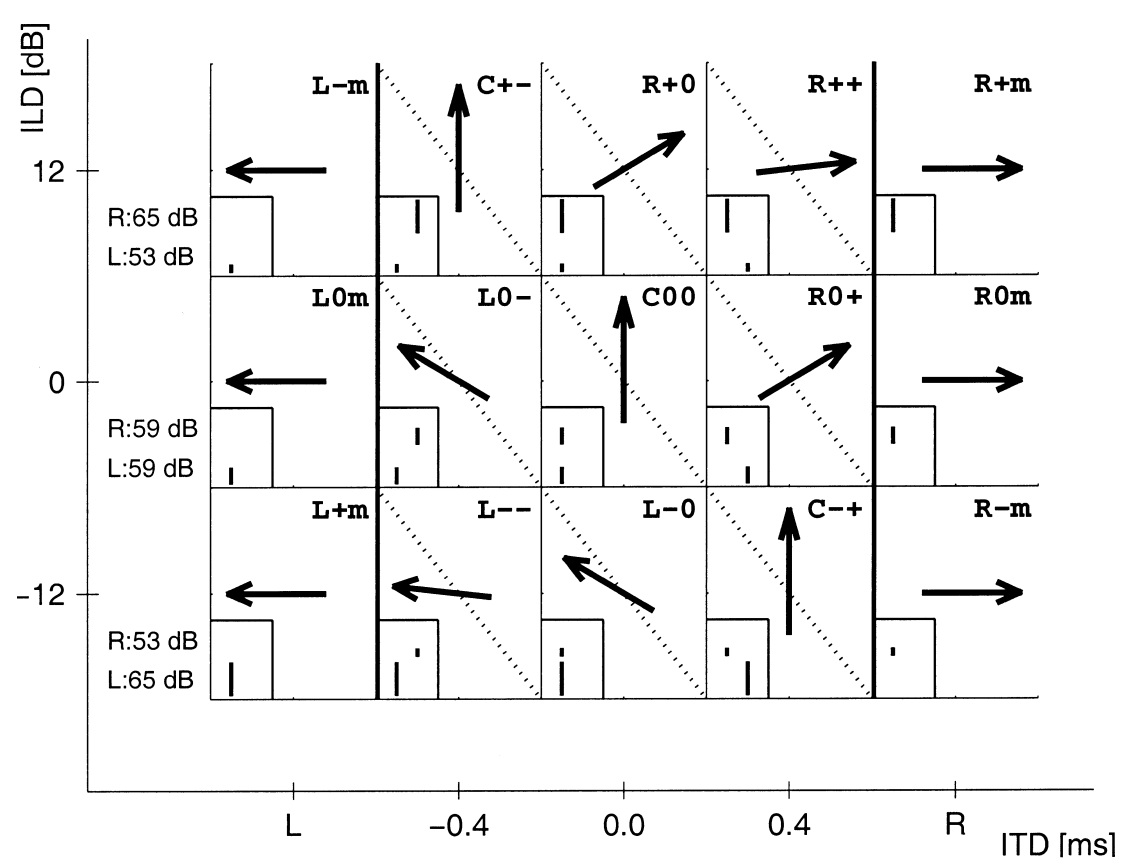

Fig. 1. Naming convention and lateralization of the stimuli: Centrally perceived stimuli are marked with a ' $\mathrm{C}$ '. Stimuli lateralized to the left and right side are marked with ' $\mathrm{L}$ ' and ' $\mathrm{R}$ ', respectively. For the monaural stimuli the second character indicates the level. For the binaural stimuli the second and third character denote the sign of ILD and ITD, respectively. Arrows point into the approximate direction of perceived lateralization. In the lower left corner of each subplot, the corresponding binaural stimulus is depicted. The dotted lines connect stimuli eliciting similar lateralization.

for every subject. The duration of the recording session was about $3 \mathrm{~h}$ without preparation and pauses.

No contralateral masking was used during the monaural presentation. This has been used in other studies to avoid ACT (Jones and van der Poel, 1990; McPherson and Starr, 1995; Brantberg et al., 1999a). Instead, following Levine (1981) and Ito et al. (1988), left, right and binaural stimuli were presented randomly without contralateral masking. In combination with the use of insert earphones and moderate presentation levels of $\leq 65 \mathrm{~dB}$ nHL, this avoids contributions of ACT and the MER to the BD.

Before averaging, the single sweeps were filtered with a linear phase FIR bandpass with 200 taps and corner frequencies 100 and $2000 \mathrm{~Hz}$ (Granzow et al., 2001). An iterated weighted average of the filtered sweeps was computed for all subjects and stimulus conditions. The optimal weighting of a sweep is known to be the inverse power of its noise. As an approximation the inverse power of the measured signal is widely used. The time course of the noise in every sweep is estimated more accurately by subtracting the weighted average from every sweep. The inverse powers of these modified sweeps from which the signal part is eliminated serve as weightings for the next iteration step in the iterative weighted averaging method. The residual noise of the averages was computed as the standard error $\sigma$ across sweeps (Riedel et al., 2001).

\subsection{Binaural interaction}

The binaural interaction was computed in terms of the $\mathrm{BD}=\mathrm{B}-(\mathrm{L}+\mathrm{R})$. This was done channel-wise and sample by sample. The six monaural stimuli were chosen to allow for the computation of the BD for all nine binaural stimulus conditions. For the stimuli with ITD the monaural response of the lagging ear was digitally delayed by the ITD before computing the BD. For the stimuli with ILD the monaural stimuli with the corresponding level were used, e.g., $\mathrm{BD}_{\mathrm{C}+-}=\mathrm{C}+-$ $-\left(\mathrm{L}-\mathrm{m}+\mathrm{R}^{\prime}+\mathrm{m}\right)$ with $\mathrm{R}^{\prime}+\mathrm{m}$ being the delayed version of $\mathrm{R}+\mathrm{m}$. All nine BDs were computed from stimulus triplets which had been recorded quasi-simultaneously, i.e., during the same measurement run. This avoids artifacts in the BD components due to long term changes of the recording conditions or subject's state. The residual noise of the $\mathrm{BD}$ was estimated as the square root of the summed variances of the three measurements, e.g.: $\sigma_{\mathrm{BD}_{\mathrm{C} 00}}=\left(\sigma_{\mathrm{C} 00}^{2}+\sigma_{\mathrm{L} 0 \mathrm{~m}}^{2}+\sigma_{\mathrm{R} 0 \mathrm{~m}}^{2}\right)^{1 / 2}$, assuming that $\mathrm{C} 00, \mathrm{~L} 0 \mathrm{~m}$ and $\mathrm{R} 0 \mathrm{~m}$ are statistically independent.

\subsection{Peak identification}

To increase the accuracy of amplitude and latency measurements, data were interpolated by a factor of 10 , i.e., they were upsampled to convert the sampling rate from 10 to $100 \mathrm{kHz}$. This was accomplished by 
zero-padding in the spectral domain which in the time domain corresponds to a convolution with a sinc-function. Since the original analog signal was band-limited to frequencies below $2 \mathrm{kHz}$, a perfect interpolation was possible.

Peaks in the interpolated signal were identified by a sign change in its derivative. For baseline-to-peak measurements peaks with voltages $V_{\mathrm{bp}}$ smaller than $2 \sigma$ were not regarded as significant and hence were discarded. In ABR measurements amplitude histograms of single sweeps show very good approximations of Gaussian distributions. We therefore interpret the residual noise as the standard error of the Gaussian measurement error $\sigma$ (Granzow et al., 2001; Riedel et al., 2001). With a confidence of $95 \%$ the true evoked potential is then in the interval $[V-2 \sigma V+2 \sigma]$ with $V$ being the measured potential. For peak-to-peak measurements peaks with voltages $V_{\text {pp }}$ greater than $\sqrt{2} 2 \sigma$ were accepted. The additional factor of $\sqrt{2}$ is due to the fact that the variances of both peaks in the pair add up. Latency errors were estimated from the amplitude errors and the curvature of the peaks according to Hoth (1986).

Automatic labelling of the peaks was consistent for wave $\mathrm{V}$ in the monaural and binaural stimulus conditions. Here, latencies and amplitudes of wave $\mathrm{V}$ were determined for all 12 subjects and all 15 stimulus conditions. Amplitudes were measured baseline-to-peak for two reasons: first, the 5-ms baseline containing 50 samples is well defined, second, peak-to-peak measurements $\mathrm{V}-\mathrm{VI}^{\prime}$ would yield erroneous amplitudes for the subjects exhibiting muscular artifacts: in 3 out of 12 subjects muscle artifacts with latencies from $8-12 \mathrm{~ms}$ in channels A1 and A2 interfere with wave $\mathrm{VI}^{\prime}$ at a latency of about $8 \mathrm{~ms}$.

In the case of the $\mathrm{BD}$, the components were labelled

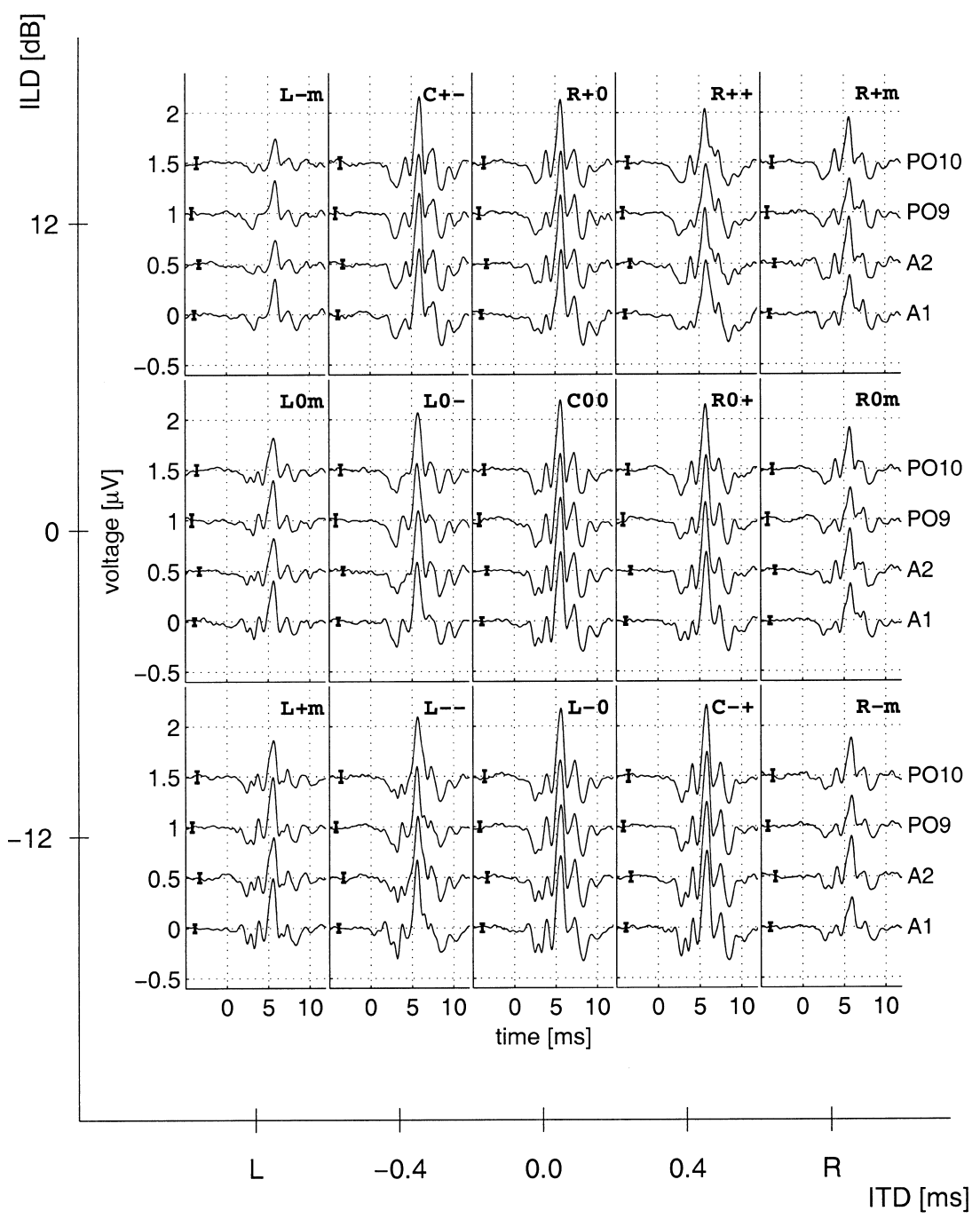

Fig. 2. ABR for the 15 stimulus conditions, 9 binaural and 6 monaural, for one subject (dj). Stimuli are arranged as in Fig. 1. Plot offset between the channels of $0.5 \mu \mathrm{V}$. Error bars indicate \pm 3 S.E.M. $( \pm 3 \sigma)$. 
manually due to their larger variability. The convention introduced by Ito et al. (1988) (see their figure 1) was adopted. The first main component of the $\mathrm{BD}$ is the negative wave DN1 preceded by a smaller positive wave labelled DP1. DN1 corresponds to the $\beta$-wave described by Levine (1981). BD amplitudes were measured peak-to-peak from DP1 to DN1 because the baseline of the $\mathrm{BD}$ shows larger fluctuations than for the monaural and binaural responses. Since the latencies of DP1 and DN1 do not deviate more than $0.5 \mathrm{~ms}$ from the latency of the binaural wave $\mathrm{V}$ there is no interference with muscular artifacts for these BD components. Latencies of the larger DN1 component were analyzed.

\section{Results}

Fig. 2 shows the recordings for all stimulus conditions, all channels and one subject. The stimuli are arranged as in Fig. 1. The binaural responses exhibit considerably larger amplitudes than the monaural responses. The highest values of wave $\mathrm{V}$ amplitude, denoted as $A_{\mathrm{V}}$, are reached for the diotic ( $\left.\mathrm{C} 00\right)$ and for the antagonistic stimuli $(\mathrm{C}+-$ and $\mathrm{C}-+)$. These stimuli are perceived more or less in the center of the head. With growing lateralization, either due to ILD or ITD, $A_{\mathrm{V}}$ decreases about the same amount. A further amplitude reduction is observed for the more lateralized synergistic stimuli. Fig. 3 shows the dependence of wave

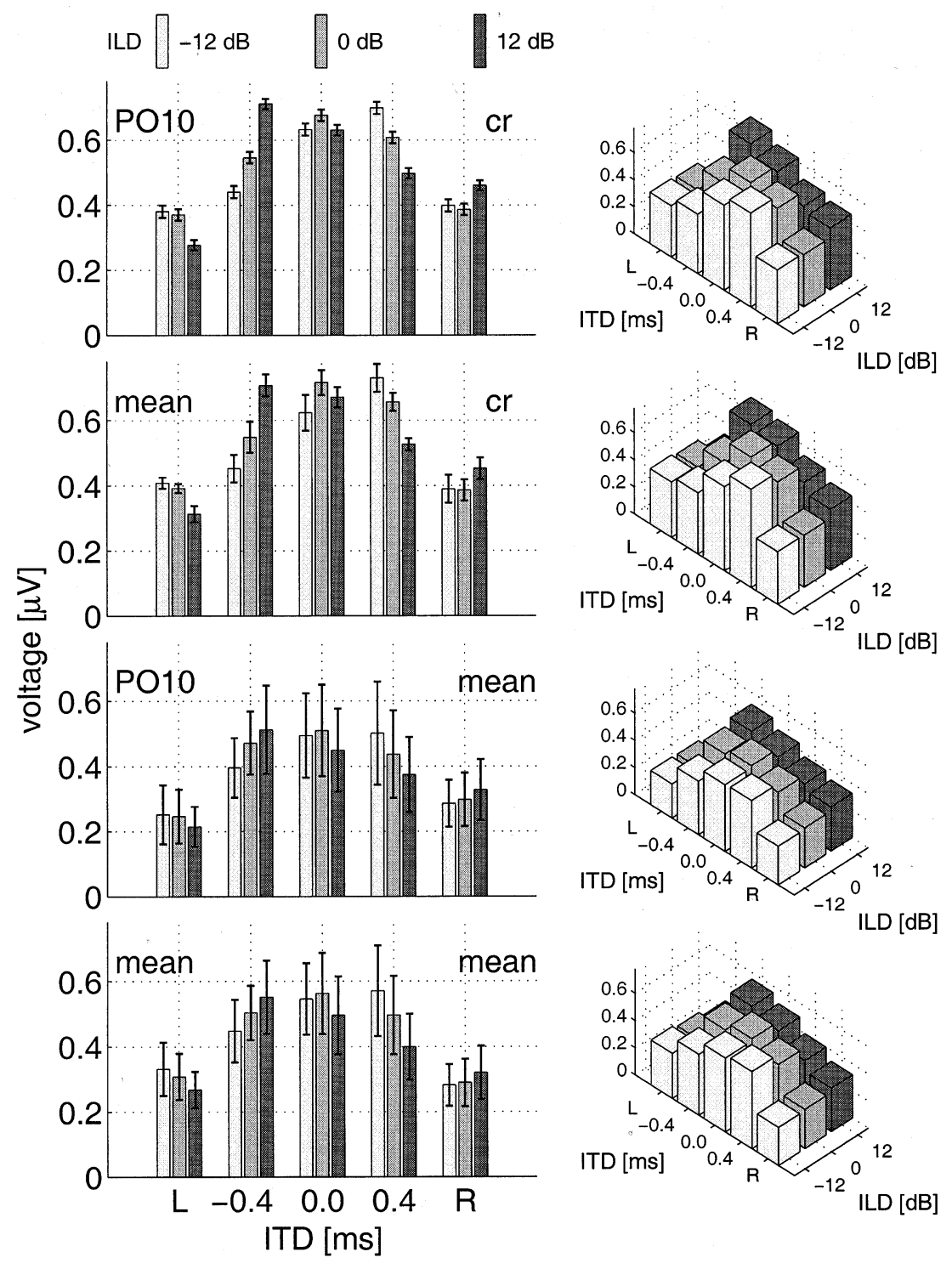

Fig. 3. Amplitudes of wave V as function of ILD and ITD. Left column: Two-dimensional representation. Brightness of the bars codes the ILD: bright $-12 \mathrm{~dB}$, gray $0 \mathrm{~dB}$ and dark $12 \mathrm{~dB}$. Right column: corresponding three-dimensional representation. Top row: Data for channel PO10 and subject cr, error bars indicate the intraindividual standard error $\sigma$. Second row: Data for mean over channels and subject cr. Third row: Data for channel PO10 and mean over subjects, error bars show interindividual standard deviations. Bottom row: Data for mean over channels and subjects. 
$\mathrm{V}$ amplitude $A_{\mathrm{V}}$ on the stimulus parameters. In the left column, $A_{\mathrm{V}}$, including error bars, is plotted two-dimensionally. The ILD is coded by different gray-scale values. In the right column, the same data are replotted three-dimensionally to illustrate $A_{\mathrm{V}}$ as a function of ILD and ITD, i.e., over the plane spanned by these parameters.

In the upper row, data for a single channel (PO10) and a single subject (cr) are plotted. As in the previous figure, the highest amplitudes are observed for the centrally perceived stimuli. This can be seen as a 'ridge' along the central diagonal in the three-dimensional graph. $A_{\mathrm{V}}$ decreases with increasing lateralization. Antagonistic stimuli have significantly higher amplitudes than synergistic stimuli, there is no overlap of the small intraindividual standard errors. The second row shows the mean over the four channels for the same subject. The third row depicts the mean over 12 subjects for channel PO10 and the bottom row the mean over channels and subjects. Apparently, interindividual standard deviations (third and fourth row) are larger than intraindividual standard errors (first and second row). This is due to the large variance of ABR across subjects. However, the differences obtained for different stimulus conditions are similar for all subjects. To reveal those differences, a signed Wilcoxon rank test was performed for all pairs of binaural stimuli. Table 1 (upper right triangle) summarizes the results for the amplitudes from the bottom row (mean over channels). Stimuli are rearranged in three groups according to their lateralization: (i) three central stimuli: $\mathrm{C} 00, \mathrm{C}-+$, and $\mathrm{C}+-$, (ii) four stimuli either lateralized by ILD or ITD: $\mathrm{L}-0$, $\mathrm{L} 0-, \mathrm{R}+0, \mathrm{R} 0+$, for convenience called the lateralized stimuli, and (iii) two synergistic stimuli: $\mathrm{L}--$ and $\mathrm{R}++$. Three significance levels were tested: significant differences for $\alpha=0.05,0.01$ and 0.001 are marked by '*', '**' and '***', respectively, 'ns' means not significant. There are no significant amplitude differences

Table 1

Differences between amplitudes (upper right triangle) and latencies (lower left triangle) of wave $\mathrm{V}$ for all pairs of binaural stimulus conditions as revealed by signed Wilcoxon rank tests across all subjects

\begin{tabular}{llllllllll}
\hline & $\mathrm{C} 00$ & $\mathrm{C}-+$ & $\mathrm{C}+-$ & $\mathrm{L}-0$ & $\mathrm{~L} 0-$ & $\mathrm{R}+0$ & $\mathrm{R} 0+$ & $\mathrm{L}--$ & $\mathrm{R}++$ \\
\hline $\mathrm{C} 00$ & - & $\mathrm{ns}$ & $\mathrm{ns}$ & $\mathrm{ns}$ & $* *$ & $* * *$ & $* * *$ & $* *$ & $* * *$ \\
$\mathrm{C}-+$ & $* * *$ & - & $\mathrm{ns}$ & $\mathrm{ns}$ & $* *$ & $* *$ & $* * *$ & $* *$ & $* * *$ \\
$\mathrm{C}+-$ & $* * *$ & $\mathrm{~ns}$ & - & $\mathrm{ns}$ & $* * *$ & $* * *$ & $* *$ & $* *$ & $* * *$ \\
$\mathrm{~L}-0$ & $*$ & $* * *$ & $* * *$ & - & $*$ & $*$ & $* *$ & $* * *$ & $* * *$ \\
$\mathrm{~L} 0-$ & $* * *$ & $* *$ & $* * *$ & $* * *$ & - & $\mathrm{ns}$ & $\mathrm{ns}$ & $*$ & $* * *$ \\
$\mathrm{R}+0$ & $*$ & $* * *$ & $* * *$ & $*$ & $* * *$ & - & $\mathrm{ns}$ & $\mathrm{ns}$ & $* * *$ \\
$\mathrm{R} 0+$ & $* * *$ & $* * *$ & $* *$ & $* * *$ & $\mathrm{~ns}$ & $* * *$ & - & $*$ & $* * *$ \\
$\mathrm{~L}--*$ & $* *$ & $* * *$ & $\mathrm{~ns}$ & $* *$ & $* *$ & $\mathrm{~ns}$ & - & $*$ \\
$\mathrm{R}++$ & $\mathrm{ns}$ & $* * *$ & $* * *$ & $\mathrm{~ns}$ & $* * *$ & $\mathrm{~ns}$ & $* * *$ & $*$ & - \\
\hline
\end{tabular}

Average data over channels (lower right panel in Fig. 4) were used for analysis. Three significance levels were tested: $* \alpha<0.05$, ${ }^{* *} \alpha<0.01,{ }^{* * *} \alpha<0.001$, ns stands for not significant. within the central group. With the exception of $\mathrm{L}-0$ all lateralized and synergistic stimuli have significantly different (smaller) wave $\mathrm{V}$ amplitudes than the central stimuli. There are also significant differences between the groups of lateralized and synergistic stimuli. With one exception $(\mathrm{L}--/ \mathrm{R}+0)$ amplitudes of the synergistic stimuli are significantly smaller than amplitudes of the lateralized stimuli.

Due to the high curvature of the peaks, individual latencies of wave $\mathrm{V}$ can be determined with high accuracy. Intraindividual standard latency errors for all subjects and stimulus conditions vary between 0.016 and $0.069 \mathrm{~ms}$, mean $0.026 \mathrm{~ms}$. The interindividual standard errors of wave $\mathrm{V}$ latencies are about an order of magnitude larger and vary between 0.19 and $0.29 \mathrm{~ms}$, mean $0.23 \mathrm{~ms}$. Fig. 4 shows the mean amplitude of wave $\mathrm{V}$ $\left(A_{\mathrm{V}}\right)$ as function of the mean latency of wave $\mathrm{V}\left(t_{\mathrm{V}}\right)$, for all channels as well as for the mean over channels. In addition, the lower left triangle in Table 1 shows the results of the signed Wilcoxon rank test for all pairs of binaural stimulus conditions to reveal the latency differences. Latencies of the antagonistic stimuli are significantly longer than those for all other binaural stimuli. The shortest latencies are observed for the synergistic stimuli and those with ITD $=0 \mathrm{~ms}$. The monaural stimuli exhibit the usual inverse relation between latency and amplitude: with increasing level $A_{\mathrm{V}}$ increases while $t_{\mathrm{V}}$ decreases. For the binaural stimuli with non-vanishing ITD this relation is inverted: The synergistic stimuli $\mathrm{L}--$ and $\mathrm{R}++$ show shortest latencies and smallest amplitudes. With decreasing lateralization both amplitude $A_{\mathrm{V}}$ and latency $t_{\mathrm{V}}$ increase.

Fig. 5 illustrates the computation of the BD from the binaural and monaural responses for one subject, one channel and diotic stimulation. The error bars show \pm 3 standard errors corresponding to a $99.7 \%$ confidence interval for Gaussian measurement errors. Filled triangles indicate extrema whose peak-to-peak voltages $V_{\mathrm{pp}}$ exceed $\sqrt{2} 3 \sigma$. Open triangles denote extrema with $V_{\text {pp }}>\sqrt{2} 2 \sigma$ that are significant only at a $95 \%$ level. This convention is maintained for all following figures.

Standard errors of monaural and binaural responses are of comparable size since they were averaged by the same number of sweeps $(10000)$. The standard errors of the composed responses $\sigma_{\mathrm{L} 0 \mathrm{~m}+\mathrm{R} 0 \mathrm{~m}}$ and $\sigma_{\mathrm{BD}_{\mathrm{C} 00}}$ are higher by a factor of about $\sqrt{2}$ and $\sqrt{3}$, respectively. This reflects the addition of the variances when adding or subtracting responses. For waves V and VI the binaural response has a slightly shorter latency than the monaural responses and their sum. The BD components DP1 and DN1 are associated with the rising and falling slope of wave $\mathrm{V}$, respectively. Analogously, but not as clear as for wave $\mathrm{V}$, the BD components DP2 and DN2 can be associated with the rising and falling slope of wave VI, respectively. 


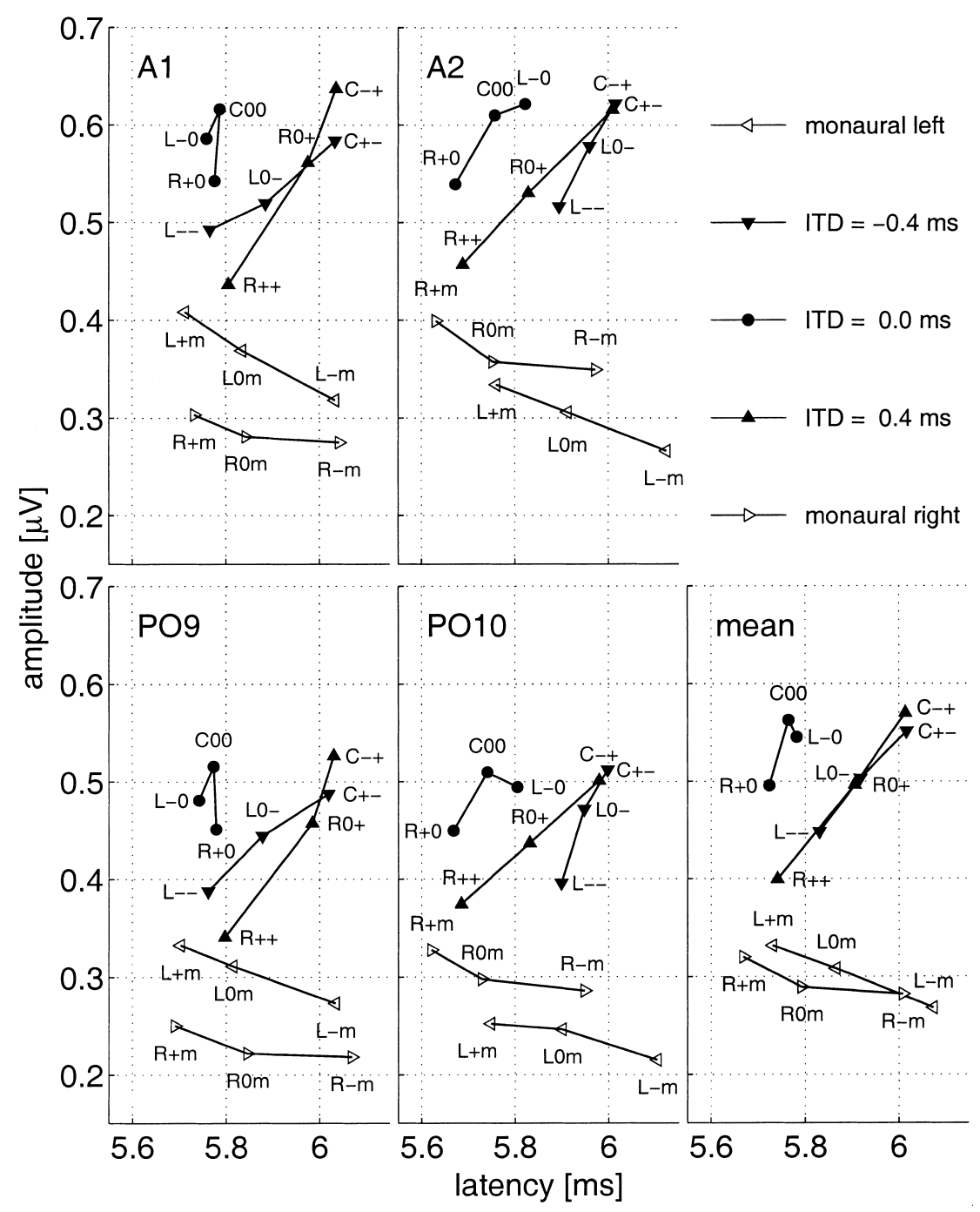

Fig. 4. Amplitude of wave $\mathrm{V}$ as function of wave $\mathrm{V}$ latency, mean over subjects. The subplots are for different channels and mean over channels. Data belonging to the same ITD are connected with lines, stimulus names are according to Fig. 1.

Fig. 6 compares the BD for all subjects in the diotic stimulus condition. Interindividual differences are mainly due to differences in residual noise level. Subjects with high residual noise level, e.g., ib and channel A2 from mk, do not show systematic BD components. Muscular artifacts in the mastoidal channels are reduced by the difference operation, but are still observable for subjects jd, kt and ow. However, the BD waves DP1 and DN1 are not affected by these artifacts. Subjects with low-noise level, e.g., cr, dj, hr and kw show a systematic BD with clear components DP1 and DN1. A DP2-DN2 complex is less reliably found, it exists for subject jo, for other subjects only in some channels. DP2 is better identifiable than DN2. Typical is the Vshaped DP1-DN1-DP2 complex.

In contrast to the monaural and binaural responses peak-to-peak measurements of amplitudes are preferable for BD due to the low SNR and poorly defined baseline. A pair of consecutive BD components is considered as significant if its peak-to-peak value $V_{\mathrm{pp}}$ exceed $\sqrt{2} 2 \sigma_{\mathrm{BD}}$ for the channel and subject considered, i.e., if its $\mathrm{SNR}$ is $\geq 6 \mathrm{~dB}$.

Table 2 summarizes amplitudes, residual noise and SNR for wave V and BD wave DP1-DN1 for all subjects. Data are mean values over the four channels for diotic stimulation. Due to the quasi-simultaneous measurement of the monaural and binaural responses the residual noise $\sigma$ is nearly constant for all stimulus conditions. Data are sorted according to the SNR of the BD component DP1-DN1 which varies between 7.4 and $15.2 \mathrm{~dB}$. Higher wave V SNRs do not necessarily entail higher SNRs of BD wave DP1-DN1. The standard error of DP1-DN1 is roughly a factor $2.4 \approx \sqrt{6}$ higher than the standard error of wave V. This can be explained by the following consideration: the addition and subtraction of responses to yield the BD contrib- 


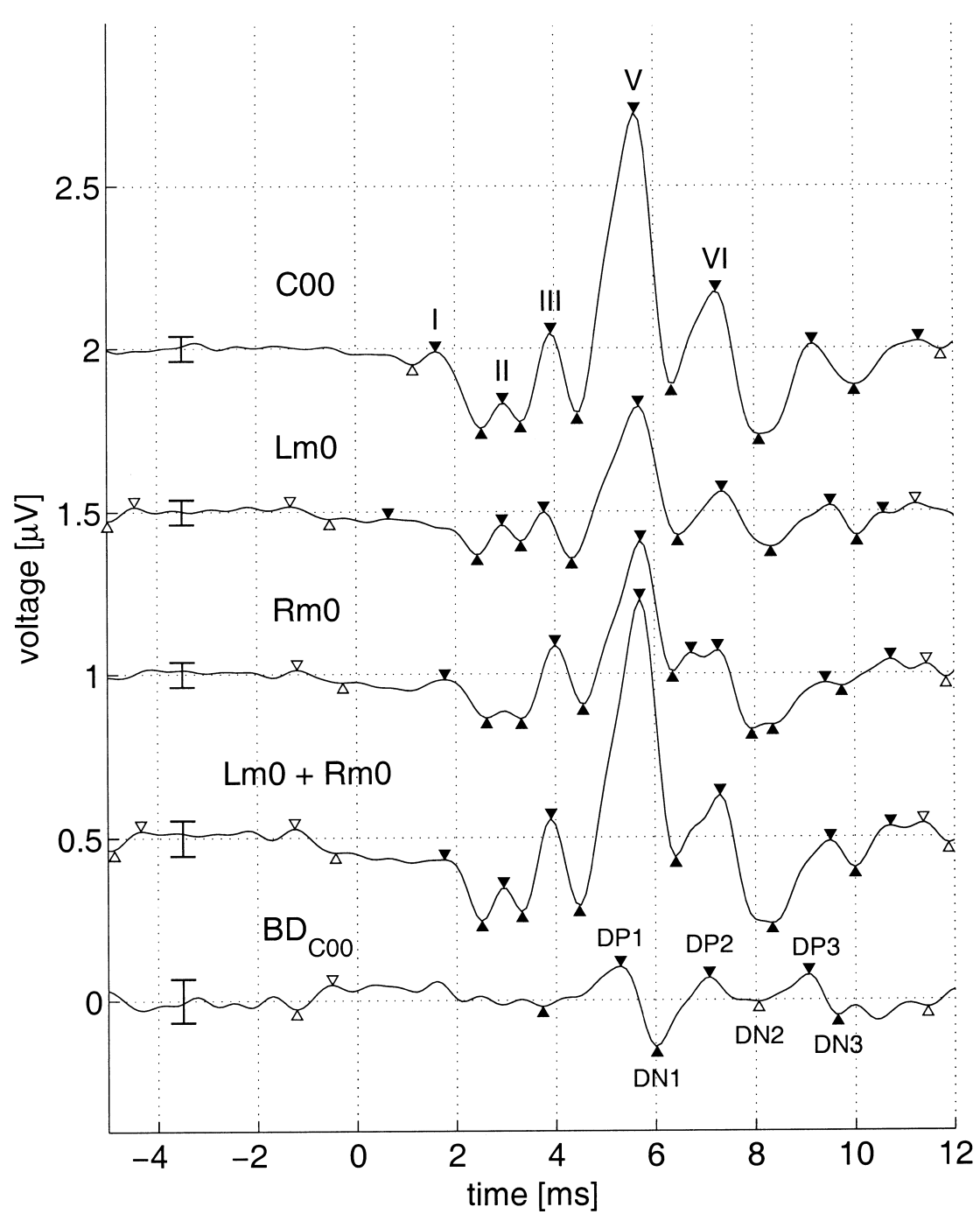

Fig. 5. Derivation of the BD for subject dj to diotic stimulation, channel A2. Top row: response to the binaural stimulus with Jewett peaks, second row: response to the monaural left stimulus, third row: response to the monaural right stimulus, fourth row: sum of the monaural responses, Botton row: BD with nomenclature of peaks. The voltage offset between adjacent curves is $0.5 \mu \mathrm{V}$. Error bars indicate \pm 3 S.E.M. $( \pm 3 \sigma)$. Open triangles: $V_{\mathrm{pp}} \geq \sqrt{2} 2 \sigma$, filled triangles: $V_{\mathrm{pp}} \geq \sqrt{2} 3 \sigma$.

utes a factor $\sqrt{3}$, another factor $\sqrt{2}$ is produced by the peak-to-peak measurement in the case of the BD compared to the baseline-to-peak measurement for wave $\mathrm{V}$.

Fig. 7 shows the dependence of average BD amplitudes $A_{\mathrm{DP} 1-\mathrm{DN} 1}$ on stimulus parameters. In the left column, the BD amplitude, DP1-DN1, including error bars, is plotted two-dimensionally. The ILD is coded by different gray-scale values. In the right column the same data are replotted three-dimensionally to illustrate $A_{\mathrm{DP} 1-\mathrm{DN} 1}$ as function over the parameter plane spanned by ILD and ITD.

In the upper row, data from subject $\mathrm{dj}$, channel $\mathrm{A} 2$, are presented. The same systematic dependence of the $\mathrm{BD}$ amplitude on stimulus conditions as for $\mathrm{ABR}$ wave $\mathrm{V}$ (see Fig. 3) is found: central stimuli (C00, C+- and $\mathrm{C}-+)$ exhibit the highest amplitudes. Except for $A_{\mathrm{R} 0+}>A_{\mathrm{C}-+}$, all stimuli lateralized only by ILD or
ITD ( $\mathrm{L}-0, \mathrm{~L} 0-$ and $\mathrm{R}+0$ ) show smaller amplitudes. A further amplitude reduction of DP1-DN1 is seen for the synergistic stimuli $\mathrm{L}--$ and $\mathrm{R}++$. However, the BDs exhibit larger intraindividual standard errors than the binaural responses. In the second row, the mean over channels for the same subject is shown. The above-mentioned exception disappeared and the systematic dependency of BD amplitude and lateralization is clearly visible in the 'ridge' for the central stimuli in the three-dimensional plot. In the third and fourth row, average data over subjects for channels A2 and the mean over channels, respectively, are drawn. As in the upper two rows increasing amplitudes are found with decreasing lateralization. Interindividual standard deviations of the BDs are in the same order of magnitude as intraindividual standard errors. To evaluate the significance of the differences in BD amplitude, signed Wil- 


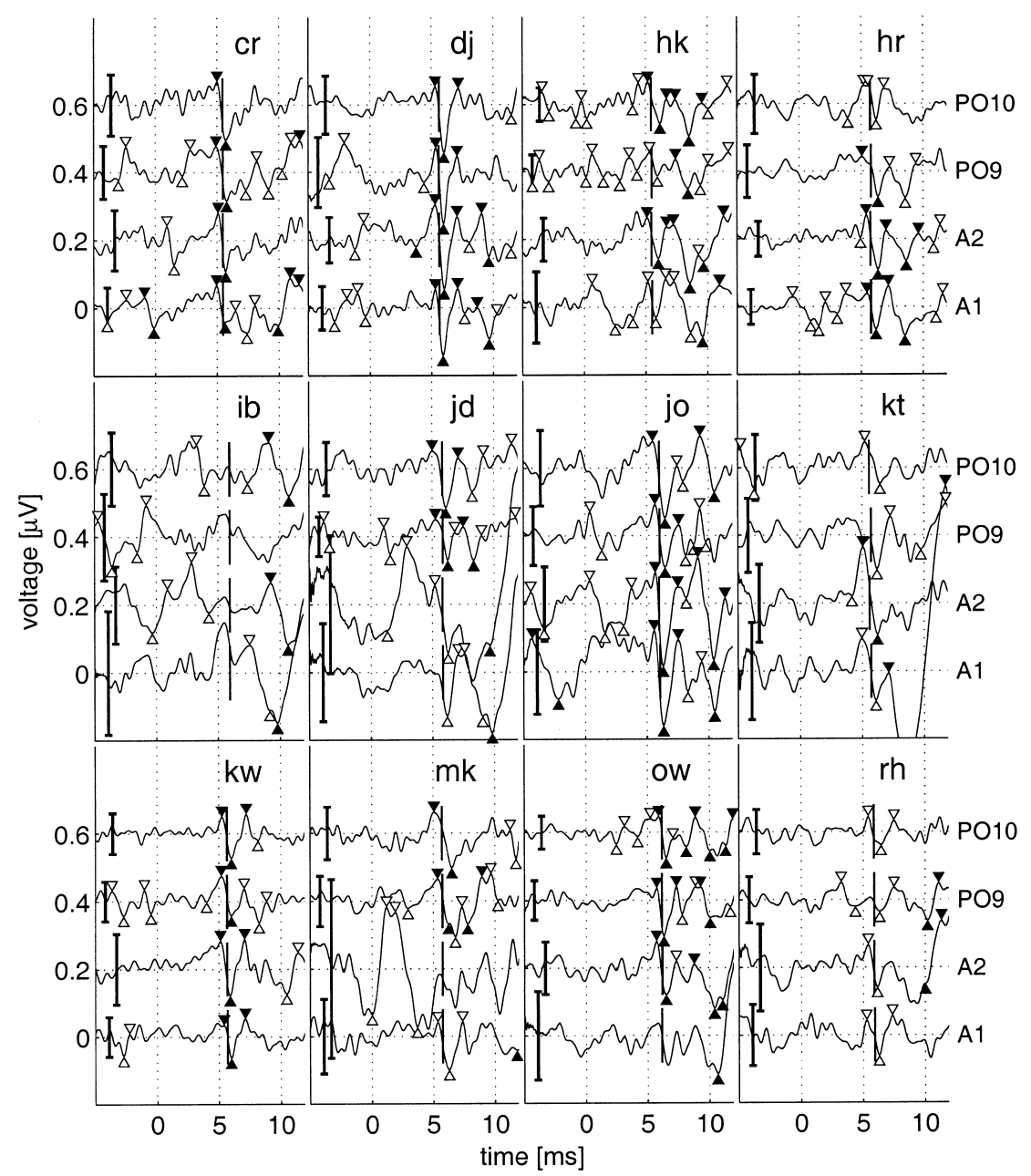

Fig. 6. BDs for 12 subjects and 4 channels. The voltage offset between adjacent curves is $0.2 \mu \mathrm{V}$. Error bars indicate \pm 3 S.E.M. Vertical lines show the latency of wave $\mathrm{V}$ in the corresponding binaural potentials. Open triangles: $V_{\mathrm{pp}} \geq \sqrt{2} 2 \sigma$, filled triangles: $V_{\mathrm{pp}} \geq \sqrt{2} 3 \sigma$.

Table 2

Amplitude A, residual noise $\sigma$ and SNR of ABR wave V and BD wave DP1-DN1 for all subjects

\begin{tabular}{|c|c|c|c|c|c|c|}
\hline \multirow[t]{2}{*}{ Subject } & \multicolumn{3}{|c|}{ ABR wave $\mathrm{V}$} & \multicolumn{3}{|c|}{ BD wave DP1-DN1 } \\
\hline & $A(\mu \mathrm{V})$ & $\sigma(\mathrm{nV})$ & SNR (dB) & $A(\mu \mathrm{V})$ & $\sigma(\mathrm{nV})$ & $\operatorname{SNR}(\mathrm{dB})$ \\
\hline $\mathrm{dj}$ & 0.69 & 15.4 & 33.2 & 0.21 & 37.7 & 15.2 \\
\hline ow & 0.48 & 15.2 & 30.4 & 0.14 & 37.1 & 13.7 \\
\hline jo & 0.65 & 20.8 & 29.9 & 0.24 & 50.9 & 13.4 \\
\hline $\mathrm{hr}$ & 0.55 & 12.9 & 33.4 & 0.12 & 31.7 & 12.1 \\
\hline cr & 0.71 & 15.4 & 25.6 & 0.15 & 37.7 & 11.7 \\
\hline $\mathrm{mk}$ & 0.42 & 25.5 & 32.8 & 0.16 & 62.0 & 11.7 \\
\hline kw & 0.62 & 13.5 & 33.5 & 0.12 & 33.1 & 11.5 \\
\hline jd & 0.72 & 23.6 & 30.7 & 0.16 & 57.0 & 10.7 \\
\hline $\mathrm{hk}$ & 0.33 & 12.9 & 28.5 & 0.10 & 31.6 & 10.5 \\
\hline kt & 0.51 & 22.5 & 26.9 & 0.19 & 54.7 & 10.2 \\
\hline $\mathrm{rh}$ & 0.47 & 16.9 & 29.1 & 0.10 & 41.5 & 7.4 \\
\hline $\mathrm{ib}$ & 0.61 & 25.6 & 27.7 & - & 62.7 & - \\
\hline Mean & 0.56 & 18.4 & 30.1 & 0.15 & 44.8 & 11.8 \\
\hline
\end{tabular}

Mean over channels, diotic stimulation. 
coxon rank tests were performed for all pairs of binaural stimulus conditions. In the upper right triangle of Table 3 the test results are shown for the mean data over channels (bottom row in Fig. 7).

Due to the smaller SNR of the BD in comparison to the binaural responses there are fewer significant differences as in the binaural case. Compared to the diotic stimulus condition (C00) BD amplitudes of the stimuli lateralized by the ITD (L0- and R0+) are significantly smaller, BD amplitudes of the stimuli with ILD (L-0 and $\mathrm{R}+0$ ) do not show a significant amplitude decrement. Except for the pair $\mathrm{C} 00$ and $\mathrm{L}--$, BD ampli- tudes of the central stimuli are significantly higher than for the synergistic stimuli.

Fig. 8 shows the mean $\mathrm{BD}$ amplitude, $A_{\mathrm{DP} 1-\mathrm{DN} 1}$, as function of the mean latency of BD wave DN1 $\left(t_{\mathrm{DN} 1}\right)$ for all channels as well as for the mean over channels, in a similar fashion as Fig. 4. The mean intraindividual standard latency error of $\mathrm{BD}$ wave $\mathrm{DN} 1$ averaged over all channels, subjects and stimulus conditions is 0.08 $\mathrm{ms}$, the mean interindividual standard deviation of DN1 latency amounts to $0.29 \mathrm{~ms}$. The lower left triangle in Table 3 gives the results of signed Wilcoxon rank tests for all pairs of binaural stimulus conditions to

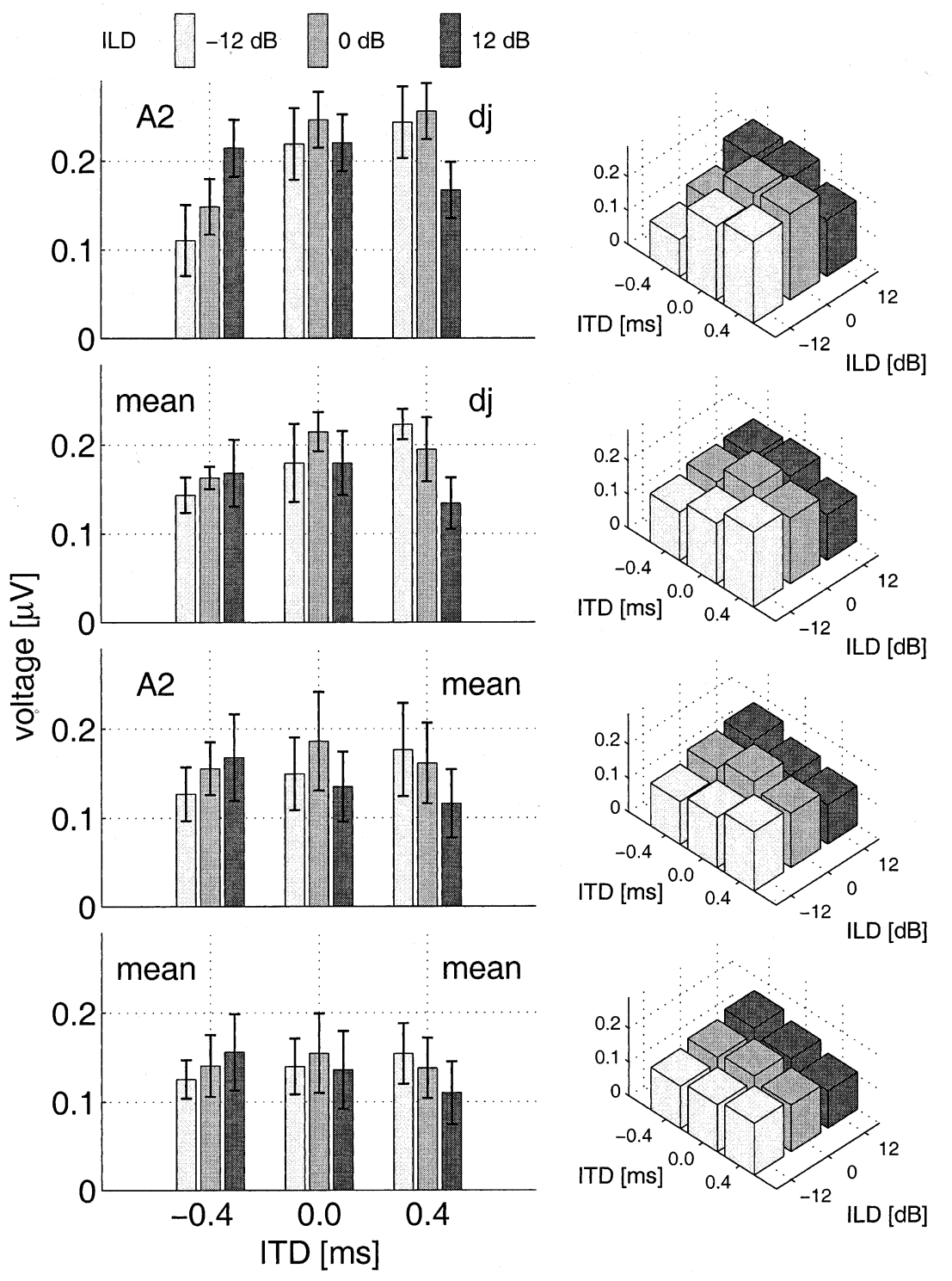

Fig. 7. Amplitudes of BD wave DP1-DN1 as function of ILD and ITD. Left column: two-dimensional representation. Brightness of the bars codes the ILD: bright $-12 \mathrm{~dB}$, gray $0 \mathrm{~dB}$ and dark $12 \mathrm{~dB}$. Right column: corresponding three-dimensional representation. Top row: Data for channel A2 and subject dj, error bars indicate the intraindividual standard error $\sigma$. Second row: Data for mean over channels and subject dj. Third row: Data for channel A2 and mean over subjects, error bars show interindividual standard deviations. Bottom row: Data for mean over channels and subjects. 
Table 3

Differences between BD amplitudes DP1-DN1 (upper right triangle) and latencies of DN1 (lower left triangle) for all pairs of binaural stimulus conditions as revealed by signed Wilcoxon rank tests across all subjects

\begin{tabular}{llllllllll}
\hline & $\mathrm{C} 00$ & $\mathrm{C}-+$ & $\mathrm{C}+-$ & $\mathrm{L}-0$ & $\mathrm{~L} 0-$ & $\mathrm{R}+0$ & $\mathrm{R} 0+$ & $\mathrm{L}--$ & $\mathrm{R}++$ \\
\hline $\mathrm{C} 00$ & - & $\mathrm{ns}$ & $\mathrm{ns}$ & $\mathrm{ns}$ & $*$ & $\mathrm{~ns}$ & $* * *$ & $\mathrm{~ns}$ & $* * *$ \\
$\mathrm{C}-+$ & $* * *$ & - & $\mathrm{ns}$ & $\mathrm{ns}$ & $\mathrm{ns}$ & $\mathrm{ns}$ & $*$ & $*$ & $* *$ \\
$\mathrm{C}+-$ & $* * *$ & $\mathrm{~ns}$ & - & $\mathrm{ns}$ & $\mathrm{ns}$ & $\mathrm{ns}$ & $\mathrm{ns}$ & $*$ & $* * *$ \\
$\mathrm{~L}-0$ & $\mathrm{~ns}$ & $* * *$ & $* * *$ & - & $\mathrm{ns}$ & $\mathrm{ns}$ & $\mathrm{ns}$ & $\mathrm{ns}$ & $* *$ \\
$\mathrm{~L} 0-$ & $* * *$ & $\mathrm{~ns}$ & $\mathrm{~ns}$ & $* * *$ & - & $\mathrm{ns}$ & $\mathrm{ns}$ & $\mathrm{ns}$ & $*$ \\
$\mathrm{R}+0$ & $\mathrm{~ns}$ & $\mathrm{~ns}$ & $* *$ & $\mathrm{~ns}$ & $*$ & - & $\mathrm{ns}$ & $\mathrm{ns}$ & $* *$ \\
$\mathrm{R} 0+$ & $* * *$ & $\mathrm{~ns}$ & $\mathrm{~ns}$ & $* * *$ & $*$ & $*$ & - & $\mathrm{ns}$ & $*$ \\
$\mathrm{~L}--$ & $* *$ & $\mathrm{~ns}$ & $\mathrm{~ns}$ & $* * *$ & $\mathrm{~ns}$ & $* *$ & $\mathrm{~ns}$ & - & $\mathrm{ns}$ \\
$\mathrm{R}++$ & $* * *$ & $\mathrm{~ns}$ & $\mathrm{~ns}$ & $* *$ & $*$ & $* * *$ & $\mathrm{~ns}$ & $\mathrm{~ns}$ & - \\
\hline
\end{tabular}

Average data over channels (lower right panel in Fig. 8) were used for analysis. Three significance levels were tested: $* \alpha<0.05$, ${ }^{* *} \alpha<0.01$ and $* * * \alpha<0.001$, ns stands for not significant. reveal significant latency differences. The stimuli with $\mathrm{ITD}=0 \mathrm{~ms}(\mathrm{C} 00, \mathrm{~L}-0$ and $\mathrm{R}+0)$ result in significantly shorter latencies than stimuli with non-vanishing ITD. In contrast to the amplitudes, the latencies of the BD wave DN1 do not clearly depend on the lateralization of the stimuli, but rather on ITD and partially on ILD. $t_{\mathrm{DN} 1}$ is mainly determined by the ITD and is, on average, $0.21 \mathrm{~ms} \approx \mathrm{ITD} / 2$ longer for stimuli with $\mathrm{ITD}= \pm 0.4 \mathrm{~ms}$ than for stimuli with $\mathrm{ITD}=0 \mathrm{~ms}$.

\section{Discussion}

High-quality recordings of $\mathrm{ABR}$ and $\mathrm{BD}$ for nine combinations of ILD and ITD were performed. Single epochs were recorded and analyzed offline allowing for an improved filtering and averaging technique as well as for an estimation of the residual noise on single-sweep basis (Granzow et al., 2001; Riedel et al., 2001). An

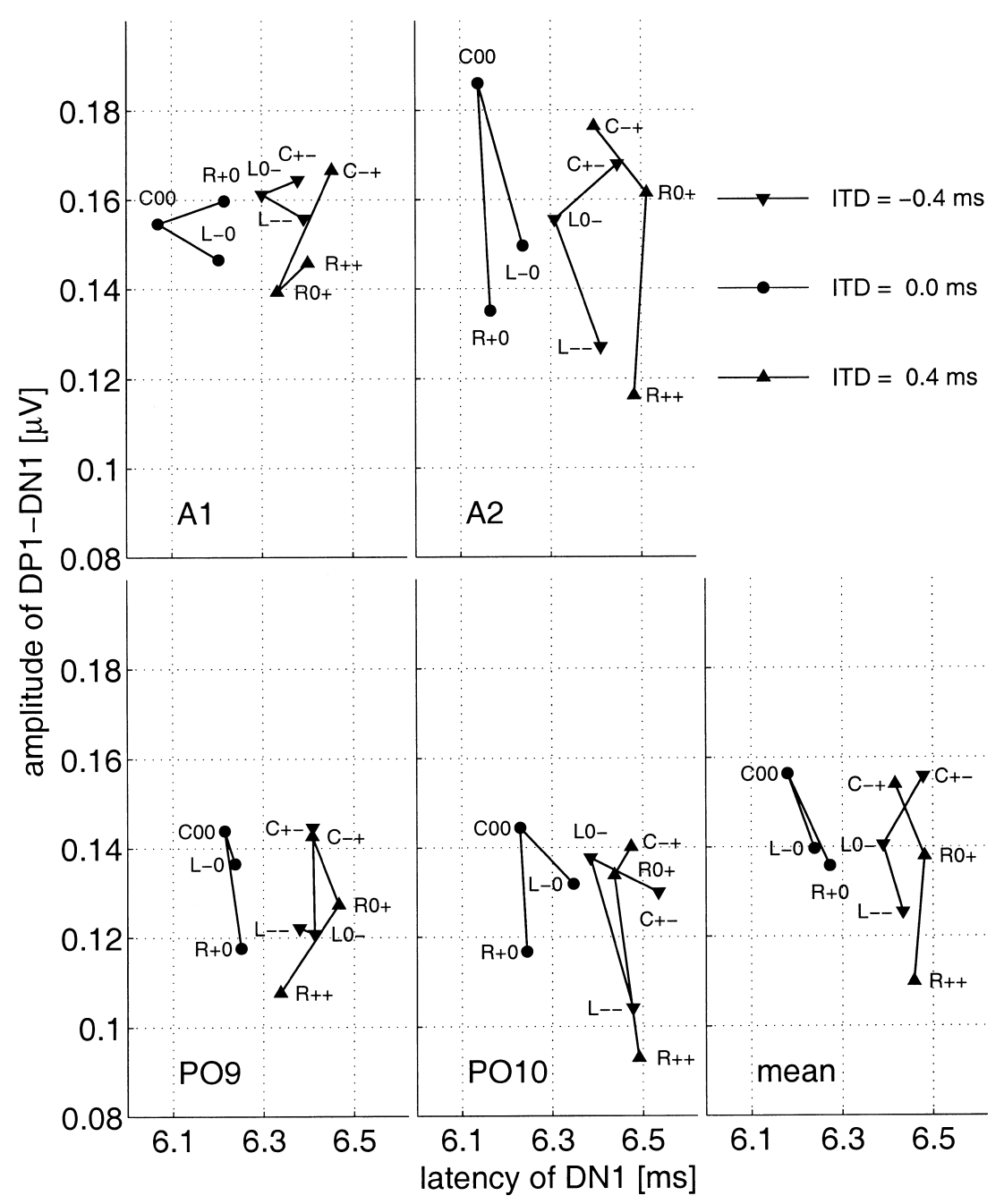

Fig. 8. BD amplitude DP1-DN1 as function of DN1 latency, mean over subjects. The subplots are for different channels and mean over channels. Data belonging to the same ITD are connected with lines, stimulus names are according to Fig. 1. 
objective SNR criterion was applied to assess the significance of the peaks. Artifact sources were ruled out by use of randomized stimulation, moderate stimulation levels and the use of insert earphones (Levine, 1981).

A systematic relation between binaural wave $\mathrm{V}$ amplitude and stimulus lateralization was clearly demonstrated for all subjects. ABR exhibit the largest amplitude for centered stimuli. With increasing lateralization the amplitude decays gradually. Wave V latency does not correlate with stimulus lateralization. It is shortest for ITD $=0$ and the synergistic stimuli and longest for the trading stimuli. Intermediate values are seen for stimuli with ITD or ILD only.

The BD component DP1-DN1 was significant in 11 out of 12 subjects in at least three of the four channels measured. BD amplitudes are maximal for diotic stimulation and decrease when ILD or ITD are introduced. However, the amplitude difference between diotic responses and responses to stimuli with ILD $= \pm 12 \mathrm{~dB}$ fail to reach significance. This finding is in agreement with the study by Furst et al. (1985) who found only a marginal decrease of the BD for ILD $= \pm 12 \mathrm{~dB}$, but in contrast to the study by McPherson and Starr (1995). They found a large difference in BD for ILD $=0 \mathrm{~dB}$ and $I L D=12 \mathrm{~dB}$, respectively, since they did not observe any BD for ILD $>8 \mathrm{~dB}$.

Our data show a significant decrease of the BD at ITD $= \pm 0.4 \mathrm{~ms}$. This is in accordance with the studies by Furst et al. (1985) and McPherson and Starr (1995). Other studies did not observe any change of the BD amplitude for ITD $= \pm 0.4 \mathrm{~ms}$ : Jones and van der Poel (1990), Brantberg et al. (1999b) found a constant DN1 up to ITD $=1 \mathrm{~ms}$. However, the number of sweeps recorded per stimulus condition used in those studies ranged from 4000 to 6400 , and in none of them a randomized stimulation paradigm was used. With the higher number of sweeps (10000) used in the present study, SNRs of the BD between about 2 and 6 , or equivalently, 7 and $15 \mathrm{~dB}$, were obtained. Therefore it is possible that the differences between the studies mentioned above result from too low SNR or from interindividual variation.

The main issue of this study was to investigate the $\mathrm{BD}$ in lateralization conditions rather than in pure ILD and ITD conditions. The BD amplitudes show a similar dependence on the stimulus parameters as observed for ABR wave $\mathrm{V}$ amplitude: The BD amplitude is smallest for the synergistic stimuli, largest for the diotic and trading stimuli. The significant differences between antagonistic and synergistic responses allow the conclusion that ILD and ITD are not processed independently in the brain stem: Let us assume that the functions $A_{\mathrm{L}}$ (ILD) and $A_{\mathrm{T}}$ (ITD) describe the dependencies of the BD amplitude on ILD and ITD, respectively. They should be symmetric functions, i.e., $A_{\mathrm{L}}$ (ILD) =
$A_{\mathrm{L}}(-\mathrm{ILD})$ and $A_{\mathrm{T}}(\mathrm{ITD})$ and $A_{\mathrm{T}}(-\mathrm{ITD})$, since the sign of the interaural disparities should not lead to different BDs. Assuming independent processing of ILD and ITD, the BD amplitude as a function of ILD and ITD would separate into two factors and could be rewritten as $A(\mathrm{ILD}, \mathrm{ITD})=A_{\mathrm{L}}(\mathrm{ILD}) A_{\mathrm{L}}(\mathrm{ITD})=A_{\mathrm{L}}(\mathrm{ILD})$ $A_{\mathrm{L}}(-\mathrm{ITD})=A(\mathrm{ILD},-\mathrm{ITD})$. Thus, synergistic and antagonistic stimuli should reveal the same responses. This clearly contradicts the experimental results. It therefore must be assumed that ILD and ITD are not processed independently in the brain stem. The experiments support the hypothesis that the lateralization angle $\gamma$ is represented by the BD ( $\gamma=0$ for centrally perceived stimuli). A possible relation of $\mathrm{BD}$ and $\gamma$ could be: $A(\mathrm{ILD}, \mathrm{ITD})=\chi(\mathrm{TIR} * \mathrm{ILD}+\mathrm{ITD})$ with TIR being the time-intensity-trading-ratio, i.e., the ILD required to compensate for the lateralization of a given ITD.

Physiological recordings in animals showed that the superior olive (SO) is the first stage of binaural interaction (e.g., Yin and Chan, 1988, 1990; Irvine, 1992). The cells in the SO were classified by the type of input they receive from the cochlear nuclei $(\mathrm{CN})$ and the medial nucleus of the trapezoid body (MNTB), (Goldberg and Brown, 1969). Excitatory-excitatory (EE)-cells in the medial SO receive excitatory input from both sides, whereas inhibitory-excitatory (IE)-cells in the lateral SO receive contralateral inhibitory input via the MNTB and ipsilateral excitatory input. EE-cells are thought to code ITDs for low frequencies performing a running cross-correlation (Jeffress, 1948) whereas IEcells are believed to code ILDs for high frequencies, thus forming the physiological basis of the duplex theory of sound localization (Rayleigh, 1907). Since in BD studies the binaural response is always found to be smaller than the sum of the monaural responses, at the first glance one would claim for binaural interaction of the IE-type. However, as pointed out by Gaumond and Psaltikidou (1991), the reduction of the binaural response could also emerge from the EE-type of interaction since EE-cells could be driven to saturation by monaural stimulation. Therefore, in EEG studies it is difficult to distinguish if the BD originates from inhibition or saturation (or both). However, there is some reasoning based on models as well as experimental data that could allow for the separation of inhibition and saturation effects.

It is generally believed that ABR to clicks mainly reflects the response to high frequencies. Therefore it can be presumed that the BD measured in response to clicks represents to a larger portion the activity of the IE-cells processing ILDs than of EE-cells processing ITDs.

Gaumond and Psaltikidou (1991) analyzed the capability of two rather simple models, one of the IE-type, the other of the EE-type, to explain the striking con- 
stancy of the amplitude ratio $A_{\mathrm{BD}} / A_{\mathrm{V}}$ independent from input level (Levine, 1981). Whereas the IE-model naturally explains the constant amplitude ratio within the EE-model $A_{\mathrm{BD}} / A_{\mathrm{V}}$ generally varies with the input level due to the compressive non-linearity. Therefore, the constant amplitude ratio $A_{\mathrm{BD}} / A_{\mathrm{V}}$ can be understood as a hint that the $\mathrm{BD}$ mainly represents binaural interaction of the IE-type.

Ungan et al. (1997) analyzed EE- and IE-models to explain the increase in latency of the BD component DN1 with increasing ITD. With a cross-correlation model of the EE-type using delay lines (Jeffress, 1948) the latency increase should be ITD/2, close to the value found in this study. However, Ungan et al. measured BDs in cat with very fine spacing of the ITD and found DN1 latency increases larger than ITD/2. Proposing a model of the IE-type, they could better explain the experimental data.

In evoked-potential studies, it was shown by means of a spatio-temporal dipole model (Scherg and von Cramon, 1985; Scherg, 1991) that the active structures at the latency of wave $\mathrm{V}$ are the SO and the lateral lemniscus (LL). This view is also supported by lesion studies from Melcher and Kiang (1996). Therefore, it must be assumed that the analysis and coding of directional information first takes place in the SO and the LL as the neural generators of the peaks in the BD. Our results indicate that a combined evaluation of interaural time and intensity cues already takes place at these stations in the human auditory pathway.

However, although significant BD peaks were shown, a further improvement in SNR seems to be highly desirable to distinguish more clearly between stimulus conditions. Of course, the number of recorded sweeps per stimulus condition could be increased in order to achieve a higher SNR. However, since measurement time grows with the square of the SNR, this is not a practical solution. Another possibility to achieve a further noise reduction is a source analysis via a dipole fit from multi-channel measurements. The interaural parameters used in this study are quite extreme, even though in the physiological range. In future studies a finer resolution and smaller values for the ITD and the ILD are desirable. Current work in progress deals with realistic stimuli using head-related transfer functions of the individual subjects.

\section{Acknowledgements}

The present work was supported by the Deutsche Forschungsgemeinschaft through the Sonderforschungsbereich Neurokognition (SFB 517). The authors would like to thank the reviewers for their helpful comments.

\section{References}

Achor, L.J., Starr, A., 1980. Auditory brain stem responses in the cat. I. Intracranial and extracranial recordings. Electroencephalogr. Clin. Neurophysiol. 48, 154-173.

Ainslie, P.J., Boston, J.R., 1980. Comparison of brain stem auditory evoked potentials for monaural and binaural stimuli. Electroencephalogr. Clin. Neurophysiol. 49, 291-302.

Brantberg, K., Fransson, P.A., Hansson, H., Rosenhall, U., 1999a. Measures of the binaural interaction component in human auditory brainstem response using objective detection criteria. Scand. Audiol. 28, 15-26.

Brantberg, K., Hansson, H., Fransson, P.A., Rosenhall, U., 1999b. The binaural interaction component in human ABR is stable within the 0 - to 1-ms range of interaural time differences. Audiol. Neurootol. 4, 88-94.

Caird, D., Klinke, R., 1983. Representation of sound frequency and laterality by units in the central nucleus of cat inferior colliculus. Exp. Brain Res. 52, 385-399.

Caird, D., Sontheimer, D., Klinke, R., 1985. Intra- and extracranially recorded auditory evoked potentials in the cat: I. Source location and binaural interaction. Electroencephalogr. Clin. Neurophysiol. $61,50-60$.

Colburn, H.S., Durlach, N.I., 1978. Models of binaural interaction. In: Carterette, E.C., Friedman, M.P. (Eds.), Handbook of Perception - Hearing. Academic Press, New York.

Cone-Wesson, B., Ma, E., Fowler, C.G., 1997. Effect of stimulus level and frequency on ABR and MLR binaural interaction in human neonates. Hear. Res. 106, 163-178.

Damaschke, J., Granzow, M., Riedel, H., Kollmeier, B., 2000. Zur Äquivalenz von interauralen Zeit- und Pegelunterschieden bei kurzen Stimuli. Z. Audiol. 39, 40-52.

Dobie, R.A., Berlin, C.I., 1979. Binaural interaction in brainstemevoked responses. Arch. Otolaryngol. 105, 391-398.

Dobie, R.A., Norton, S.J., 1980. Binaural interaction in human auditory evoked responses. Electroencephalogr. Clin. Neurophysiol. 49, 303-313.

Durlach, N.I., Colburn, H.S., 1978. Binaural phenomena. In: Carterette, E.C., Friedman, M.P. (Eds.), Handbook of Perception Hearing. Academic Press, New York.

Feddersen, W.E., Sandel, T.T., Teas, D.C., Jeffress, L.A., 1957. Localization of high-frequency tones. J. Acoust. Soc. Am. 29, 988991.

Furst, M., Levine, R.A., McGaffigan, P.M., 1985. Click lateralization is related to the $\beta$ component of the dichotic brainstem auditory evoked potentials of human subjects. J. Acoust. Soc. Am. 78, 1644-1651.

Gaumond, R.P., Psaltikidou, M., 1991. Models for the generation of the binaural difference response. J. Acoust. Soc. Am. 89, 454-456.

Gerull, G., Mrowinski, D., 1984. Brain stem potentials evoked by binaural click stimuli with differences in interaural time and intensity. Audiology 23, 265-276.

Goldberg, J.M., Brown, P.B., 1969. Response of binaural neurons of dog superior olivary complex to dichotic tonal stimuli: some physiological mechanisms of sound localization. J. Neurophysiol. 36, $157-178$.

Granzow, M., Riedel, H., Kollmeier, B., 2001. Single-sweep-based methods to improve the quality of auditory brain stem responses. Part I: Optimized linear filtering. Z. Audiol. 40, 32-44.

Gummer, A.W., Zenner, H.P., 1996. Central processing of the auditory information. In: Greger, R., Windhorst, U. (Eds.), Comprehensive human physiology. Springer, Berlin.

Hoth, S., 1986. Reliability of latency and amplitude values of auditory-evoked potentials. Audiology 25, 248-257.

Irvine, D.R., 1992. Physiology of the auditory brainstem. In: Popper, 
A.N., Fay, R.R. (Eds.), The Mammalian Auditory Pathway: Neurophysiology. Springer, New York.

Ito, S., Hoke, N., Pantev, C., Lütkenhöner, B., 1988. Binaural interaction in brainstem auditory evoked potentials elicited by frequency-specific stimuli. Hear. Res. 35, 9-20.

Jasper, H.H., 1957. The ten twenty electrode system of the international federation (Appendix). Electroencephalogr. Clin. Neurophysiol. 10, 371-375.

Jeffress, L.A., 1948. A place theory of sound localization. J. Comp. Physiol. Psychol. 41, 35-39.

Jiang, Z.D., 1996. Binaural interaction and the effects of stimulus intensity and repetition rate in human auditory brain-stem. Electroencephalogr. Clin. Neurophysiol. 100, 505-516.

Jones, S.J., van der Poel, J.C., 1990. Binaural interaction in the brainstem auditory evoked potential: evidence for a delay line coincidence detection mechanism. Electroencephalogr. Clin. Neurophysiol. 77, 214-224.

Joseph, A.W., Hyson, R.L., 1993. Coincidence detection by binaural neurons in the chick brain stem. J. Neurophysiol. 69, 11971211.

Kelly-Ballweber, D., Dobie, R.A., 1984. Binaural interaction measured behaviorally and electrophysiologically in young and old adults. Audiology 23, 181-194.

Levine, R.A., 1981. Binaural interaction in brainstem potentials of human subjects. Ann. Neurol. 9, 384-393.

McPherson, D.L., Starr, A., 1995. Auditory time-intensity cues in the binaural interaction component of the auditory evoked potentials. Hear. Res. 89, 162-171.

McPherson, D.L., Tures, C., Starr, A., 1989. Binaural interaction of the auditory brain-stem potentials and middle latency auditory evoked potentials in infants and adults. Electroencephalogr. Clin. Neurophysiol. 74, 124-130.

Melcher, J.R., Kiang, N.Y.S., 1996. Generators of the brain-stem auditory-evoked potential in cat. 3. Identified cell-populations. Hear. Res. 93, 52-71.

Mills, A.W., 1958. On the minimum audible angle. J. Acoust. Soc. Am. 30, 237-246.

Mills, A.W., 1960. Lateralization of high-frequency tones. J. Acoust. Soc. Am. 32, 132-134.
Nieuwenhuys, R., Vogel, J., van Huijzen, C., 1988. The Human Central Nervous System - A Synopsis and Atlas. Springer, Berlin.

Nordby, K., Rosness, R., Skotturi, B.C., 1982. Binaural time/intensity trading. J. Acoust. Soc. Am. 71, 132-134.

Popper, A.N., Fay, R.R., 1992. The mammalian auditory pathway: Neurophysiology. Springer, New York.

Rayleigh, L.S.J.W., 1907. On our perception of sound direction. Philos. Mag. 13, 214-232.

Riedel, H., Granzow, M., Kollmeier, B., 2001. Single-sweep-based methods to improve the quality of auditory brain stem responses. Part II: Averaging methods. Z. Audiol. 40, 62-85.

Scherg, M., 1991. Akustisch evozierte Potentiale: Grundlagen - Entstehungsmechanismen - Quellenmodell. Kohlhammer, Stuttgart.

Scherg, M., von Cramon, D., 1985. A new interpretation of the generators of BAEP waves I-V: results of a spatio-temporal dipole model. Electroencephalogr. Clin. Neurophysiol. 62, 290-299.

Semple, M.N., Aitkin, L.M., 1989. Representation of sound frequency and laterality by units in the central nucleus of cat inferior colliculus. J. Neurophysiol. 42, 1626-1639.

Sharbrough, F., Chatrian, G.E., Lesser, R.P., Lüders, H., Nuwer, M., Picton, T.W., 1991. American Electroencephalographic Society Guidelines for Standard Electrode Position Nomenclature. J. Clin. Neurophysiol. 8, 200-202.

Ungan, A., Yagcioglu, S., Özmen, B., 1997. Interaural delay-dependent changes in the binaural difference potential in cat auditory brainstem response: implications about the origin of the binaural interaction component. Hear. Res. 106, 66-82.

van Adel, B.A., Kidd, S.A., Kelly, J.B., 1999. Contribution of the commissure of Probst to binaural evoked responses in the rat's inferior colliculus: interaural time differences. Hear. Res. 130, $115-130$.

Wrege, K.S., Starr, A., 1981. Binaural interaction in human auditory brainstem evoked potentials. Arch. Neurol. 38, 572-580.

Yin, T.C., Chan, J.C., 1988. Neural mechanisms underlying interaural time sensitivity to tones and noise. In: Edelmann, G.M., Gall, W.E., Cowan, W.M. (Eds.), Auditory Function: Neurobiological Bases of Hearing. John Wiley and Sons, New York.

Yin, T.C.T., Chan, J.C.K., 1990. Interaural time sensitivity in medial superior olive of cat. J. Neurophysiol. 64, 465-488. 